\title{
IncRNA TUG1-Mediated Mir-142-3p Downregulation Contributes to Metastasis and the Epithelial-to-Mesenchymal Transition of Hepatocellular Carcinoma by Targeting ZEB1
}

\author{
${\text { Chuan } \mathrm{He}^{\mathrm{a}} \text { Zhigang Liu }{ }^{\mathrm{a}} \text { Li Jin }{ }^{\mathrm{b}} \text { Fang Zhang }{ }^{\mathrm{b}} \quad \text { Xinhao Peng }}^{\mathrm{b}}$ \\ Yaqin Xiao ${ }^{\mathrm{b}}$ Xi Wang ${ }^{\mathrm{b}}$ Qian Lyu ${ }^{\mathrm{c}}$ XiaoJun Caid
}

aDepartment of Hematology, Hematology Research Laboratory, West China Hospital, Sichuan University, Chengdu, 'Department of Oncology, Sichuan Cancer Hospital \& Institute, Chengdu, 'Department of Pathology, Nanjing First Hospital, Nanjing Medical University, Nanjing, dTraditional Chinese Medicine Academy of Heilongjiang, Harbin, China

\section{Key Words}

Lncrna TUG1 • Hepatocellular carcinoma • MiR-142-3p • ZEB1 • EMT

\begin{abstract}
Background/Aims: MicroRNA-142-3p (miR-142-3p) is dysregulated in many malignancies and may function as a tumor suppressor or oncogene in tumorigenesis and tumor development. However, few studies have investigated the clinical significance and biological function of miR-142-3p in hepatocellular carcinoma (HCC). Methods: The expression levels of taurine upregulated gene 1 (TUG1), miR-142-3p, and zinc finger E-box-binding homeobox 1 (ZEB1) were evaluated in HCC tissues and cell lines by quantitative real-time PCR. MTT and colony formation assays were used to detect cell proliferation ability, transwell assays were used to assess cell migration and invasion, and luciferase reporter assays were used to examine the interaction between the long noncoding RNA TUG1 and miR-142-3p. Tumor formation was evaluated through in vivo experiments. Results: miR-142-3p was significantly downregulated in HCC tissues, but TUG1 was upregulated in HCC tissues. Knockdown of TUG1 and upregulation of miR-142-3p inhibited cell proliferation, cell migration, cell invasion, and the epithelial-mesenchymal transition (EMT). miR-142-3p was found to be a prognostic factor of HCC, and the mechanism by which TUG1 upregulated ZEB1 was via direct binding to miR142-3p. In vivo assays showed that TUG1 knockdown suppressed cell proliferation and the EMT in nude mice. Conclusion: The results of this study suggest that the TUG1/miR-142-3p/ ZEB1 axis contributes to the formation of malignant behaviors in HCC.

C. He, Z. Liu and L. Jin contributed equally to this work.




\section{Introduction}

Hepatocellular carcinoma (HCC) is the second highest cause of cancer-related mortality worldwide due to late tumor presentation and rapid progression Siegel et al. [1, 2]. It has distinct clinical, pathological and molecular features, but the molecular mechanisms by which the biological behaviors of HCC are regulated are not fully understood.

Recently, emerging evidence has indicated that noncoding RNAs (ncRNAs) may participate in HCC pathogenesis Xiong et al. [3-7]. Integrative genomic studies have shown that about $98 \%$ of human genome transcripts are ncRNAs Mattick et al. [8-10]. As endogenous and single-stranded RNAs, microRNAs (miRNAs) have been found in diverse diseases, and it has been demonstrated that they can negatively regulate their target messenger RNAs (mRNAs) through translational suppression or mRNA degradation Ohtsuka et al. [1113]. The regulatory role of miR-142-3p has been reported in several types of cancer. For example, miR-142-3p overexpression increases the chemosensitivity of non-small cell lung cancer (NSCLC) by inhibiting high-mobility group box 1-mediated autophagy Chen et al. [14]. In addition, it can modulate osteosarcoma progression by interacting with metastasisassociated lung adenocarcinoma transcript-1 Liu et al. [15]. Some studies have examined the biological role of miR-142-3p in HCC. Xue et al. [16]. demonstrated that miR-142-3p directly downregulates Ras-related C3 botulinum toxin substrate 1 in HCC cells; Wang et al. [17]. showed that miR-142-3p and miR-142-5p synergistically suppress cell motility in HCC. However, the effects of miR-142-3p on the epithelial-mesenchymal transition (EMT) process in HCC are still unknown. This study explored the clinical significance, biological role, and underlying molecular mechanism of miR-142-3p in the development and progression of HCC.

As a group of ncRNAs, long noncoding RNAs (lncRNAs) act as competing endogenous RNAs (ceRNAs) by binding with miRNAs Yang et al. $[18,19]$. For instance, lncRNA CCAT1 promotes gallbladder cancer development by negatively modulating miRNA-218-5p Ma et al. [20]; IncRNA growth-arrest specific 5 (GAS5) inhibits tumorigenesis and enhances radiosensitivity in NSCLC by suppressing miR-135b expression Xue et al. [16].; and lncRNA H19 and highly upregulated in liver cancer (HULC) can act as ceRNAs to promote cell migration and invasion in cholangiocarcinoma Wang et al. [21]. The lncRNA taurine upregulated gene 1 (TUG1) plays a role in the progression of several human cancers by interacting with miRNAs Wang et al. [22-24]. For example, TUG1 accelerates the malignancy of pancreatic cancer through sponging miR-382 Zhao et al. [25], mediates methotrexate resistance in colorectal cancer (CRC) by interacting with miR-186 Li et al. [26], acts as an oncogene in osteosarcoma by binding with miR-335 Wang et al. [22], and improves gastric cancer progression through sponging miR-145-5p Ren et al. [24]. Thus, this study aimed to clarify the interaction between TUG1 and miR-142-3p in HCC.

In this study, the low expression level of miR-142-3p was detected in HCC tissues. Furthermore, overexpression of miR-142-3p was found to significantly repress cell metastasis and reverse the EMT formation. The results of the mechanistic experiments revealed that miR-142-3p exerted anti-oncogenic functions in HCC in a zinc finger E-boxbinding homeobox 1 (ZEB1)-dependent manner. Moreover, bioinformatics analysis revealed that TUG1 could directly bind to miR-142-3p. TUG1 was found to be highly expressed in HCC tissues and was inversely correlated with miR-142-3p expression. Additional studies confirmed that TUG1 negatively regulated miR-142-3p expression by functioning as a ceRNA. Collectively, our findings suggest that the TUG1/miR-142-3p/ZEB1 pathway contributes to the progression of HCC. 


\section{Cellular Physiology Cell Physiol Biochem 2018;48:1928-1941 \begin{tabular}{ll|l} 
DOI: 10.1159/000492517 & Ond Biochemistry 2018 The Author(s). Published by S. Karger AG, Basel \\
Published online: August 8, 2018 & \begin{tabular}{l} 
www.karger.com/cpb \\
\hline
\end{tabular}
\end{tabular}}

and Biochemistry

\section{Materials and Methods}

\section{Patients}

A total of 92 HCC tissues and matched tumor-adjacent tissues were obtained from the Department of Gastrointestinal Surgery, Shaoxing People's Hospital, Shaoxing Hospital of Zhejiang University (Shaoxing, China). Tissue specimens were conserved in liquid nitrogen for RNA isolation. No patients received immunotherapy, radiotherapy, or chemotherapy before surgery. All samples were used after informed consent was obtained.

\section{Cell culture}

For routine cell culture, HepG2, Hep3B, SMMC-7721, HCCLM3, and Bel-7402 cell lines and the L02 normal liver epithelium cell line, purchased from the Shanghai Cell Collection (Shanghai, China), were maintained in high-glucose Dulbecco's Modified Eagle's Medium (8113035; Gibco, Gaithersburg, MD, USA) medium with $10 \%$ fetal bovine serum, $100 \mathrm{U} / \mathrm{mL}$ penicillin, and $100 \mathrm{mg} / \mathrm{mL}$ streptomycin in a humidified air at $37^{\circ} \mathrm{C}$ with $5 \%$ of $\mathrm{CO}_{2}$.

\section{qRT-PCR}

Based on the manufacturer's protocols, RNA was isolated using Trizol (Invitrogen, Carlsbad, CA, USA). First-strand cDNA was synthesized using the Tianscript RT Kit (Tiangen Biotech, Beijing, China). PCR amplification for miR-142-3p, TUG1, and ZEB1 mRNA was performed with the TaqMan Human MiRNA Assay (Genecopoeia, Guangzhou, China) and SYBR Premix Ex Taq ${ }^{\mathrm{TM}}$ Kit (Takara, Shiga, Japan) using the ABI 7300 System (Applied Biosystems, Foster City, CA, USA). As reference genes, U6 was used to normalize the expression of miR-142-3p, and GAPDH was used to normalize the expression of TUG1 and ZEB1 mRNA.

\section{Migration and invasion assay}

Cell migration and invasion were measured using transwell cell culture chambers ( $8 \mu \mathrm{m}$ pore size, Corning Inc., Corning, NY, USA) with and without Matrigel (Becton Dickinson, Franklin Lakes, NJ, USA). At 48 $\mathrm{h}$ post-transfection, cells in serum-free media were placed in the upper chamber with and without $10 \mu \mathrm{g} /$ $\mathrm{mL}$ Matrigel. Medium containing 10\% bovine calf serum was added to the lower chamber. Following $48 \mathrm{~h}$ of incubation, cells remaining in the upper membrane were wiped off, whereas cells that had migrated were fixed in methanol, stained with $0.1 \%$ crystal violet, and counted under a microscope. Three independent experiments were conducted.

\section{MTT assay}

Cell viability was measured using the Cell Proliferation Reagent Kit I (MTT; Roche Applied Science, Penzberg, Germany). The SMMC-7221 and HepG2 cells were added to 96-well plates at a density of $2 \times 10^{3}$ cells per well and then cultured in six replicate wells. Cell proliferation was evaluated at 24, 48, and $72 \mathrm{~h} ; 10$ $\mu \mathrm{L}$ MTT solution was added to each well in the last $4 \mathrm{~h}$ of the incubation period. Cell viability was defined as the mean of the absorbance of the converted dye at $450 \mathrm{~nm}$.

\section{Colony formation assay}

For the colony formation assay, we added 500 cells per well to 6-well plates and cultured them in normal medium at $37^{\circ} \mathrm{C}$. After 14 days, the cells were fixed in methanol and then dyed with $0.1 \%$ crystal violet. Visible colonies were counted manually.

\section{Xenograft transplantation and immunohistochemistry}

Approximately $5.0 \times 10^{6}$ SMMC-7721 cells stably transfected with sh-TUG1 or shRNA suspended in $100 \mu \mathrm{l}$ phosphate-buffered saline were injected subcutaneously into the right side of the posterior flank of female BALB/c athymic nude mice (Department of Gastrointestinal Surgery, Shaoxing People's Hospital, Shaoxing Hospital of Zhejiang University) at 5-6 weeks of age. Tumor growth was examined every other day with a vernier caliper. Tumor volumes were calculated using the equation: $V=A \times B^{2} / 2\left(\mathrm{~mm}^{3}\right)$, where $\mathrm{A}$ is the largest diameter and B is the perpendicular diameter. After 5 weeks, all mice were sacrificed and 


\section{Cellular Physiology Cell Physiol Biochem 2018;48:1928-1941 \begin{tabular}{l|l} 
and Biochemistry Published 10.1159/000492517 & $\begin{array}{l}\text { (c) 2018 The Author(s). Published by S. Karger AG, Basel } \\
\text { www.karger.com/cpb }\end{array}$ \\
\hline
\end{tabular}}

He et al.: The Function of TUG1-miR-142-3p-ZEB1 Axis in HCC

necropsies were performed. The primary tumors were excised, paraffin-embedded, and formalin-fixed, followed by hematoxylin and eosin staining and immunostaining for Ki-67 protein expression according to the manufacturer's instructions. All animal experiments were strictly conducted in accordance with ethical standards and performed in accordance with the institutional guidelines.

\section{Western blotting}

Total proteins were collected with RIPA lysis buffer (Santa Cruz Biotechnology, Inc., Santa Cruz, CA, USA) and protein concentrations were measured with the BCA method (Beyotime, Jiangsu, China). A total of $40 \mu \mathrm{g}$ protein was subjected to $4-20 \%$ sodium dodecyl sulfate polyacrylamide gel electrophoresis (Sigma St. Louis, MO, USA), after which the proteins were electrophoretically transferred to polyvinylidene fluoride membranes (Roche, Indianapolis, IN, USA). Then, the membranes were blocked in 5\% milk, followed by incubation with primary antibodies for ZEB1 (Abcam, Cambridge, MA, USA), E-cadherin (Abcam), N-cadherin (Abcam), $\alpha$-SMA (Abcam), or Vimentin (Abcam) and subsequent incubation with the appropriate secondary antibodies (Cell Signaling Technology, Beverly, MA, USA). GAPDH (US Biological, Swampscott, MA, USA) was used as a loading control.

\section{Immunofluorescence}

HCC cells transfected with the appropriate miRNA vectors were seeded onto chamber slides and fixed in $4 \%$ paraformaldehyde for $10 \mathrm{~min}$ at room temperature. Then, cells were incubated with antibodies against E-cadherin (Abcam) or $\mathrm{N}$-cadherin (Abcam) at $4^{\circ} \mathrm{C}$ overnight. Slides were incubated with matched secondary antibodies (Invitrogen) at room temperature for $1 \mathrm{~h}$. The nucleus of HCC cells were stained with DAPI (Sigma) at room temperature for $10 \mathrm{~min}$. Fluorescence confocal images were captured using the LSM 5 Pascal Laser Scanning Microscope (Zeiss Germany, Oberkochen, Germany).

\section{Plasmid construction and dual luciferase reporter assay}

Full-length (537 base pairs) human TUG1 WT with a potential miR-142-3p binding site or MUT site was generated and fused to the luciferase reporter vector psi-CHECK-2 (Promega, Madison, WI, USA), designated pTUG1-WT or pTUG1-MUT, respectively, and amplified using the following primers: TUG1-WT forward:5'TTTAGGGTTATTGGCCAAGACGGTTCCG-3' and reverse: 5'-GGTTTGAATTATATGAATACCGGTTATATAG-3'; TUG1-MUT forward: 5'-CTTGTGAGGCTAAGGTCCTTAGGGCTG-3' and reverse: 5'-TTCTTGCATTAATGGTTAGGCCTTATGGTTTA-3'. Full-length human WT ZEB1 with a potential miR-142-3p binding site or MUT site was generated and fused to the luciferase reporter vector psi-CHECK-2 (Promega), designated pZEB1-WT or pZEB1-MUT, respectively, and amplified with the following primers: forward: 5'-AGTAGATATACAGAATATTAACTATGTG-3' and reverseprimer:5'-TCTAGGCTTATGTTTCTCTGTCTC-3';ZEB1MUT forward: 5'-ATTAGATATGAGTTATAACGATGATG-3' and reverse: 5'-TAGTACCATTAGCGGTATACCATGTC-3'. Luciferase reporter assay were performed in 96-well plates using a Dual Luciferase Reporter Assay kit (Dual-Glo Luciferase Assay System; Promega) and SpectraMax M5 instrument software (Molecular Devices, Sunnyvale, CA, USA) was used to analyze the results. Cells were co-transfected with 100 ng pZEB1-miR-1423p-UTR-WT or pZEB1-miR-142-3p-UTR-MUT and pTUG1-miR-142-3p-UTR-WT or pTUG1-miR-142-3pUTR-MUT with $50 \mathrm{nM}$ Lipofectamine 2000 (Life Technologies, Carlsbad, CA, USA). After 48 h, the luciferase assay was performed using a Dual-Luciferase Reporter Assay kit (Promega) according to the manufacturer's instructions.

\section{RIP}

RIP was performed using a RIP Kit (Thermo Fisher Scientific, Waltham, MA, USA) in accordance with the manufacturer's protocol. Cells at $80-90 \%$ confluency were scraped off and lysed in complete RIP lysis buffer. Then, $100 \mu \mathrm{L}$ whole cell extract was incubated with RIP buffer containing magnetic beads conjugated to human anti-Ago2 antibody (Cell Signaling Technology); the negative control was normal mouse IgG (Millipore) and SNRNP70 (Millipore, Stafford, VA, USA) binding to U1 was used as the positive control. The co-precipitated RNAs were detected by reverse transcription PCR. Total RNAs (input controls) and IgG were assayed simultaneously to test whether the detected signals resulted from RNAs specifically bound to Ago 2 . 


\section{Cellular Physiology Cell Physiol Biochem 2018;48:1928-1941

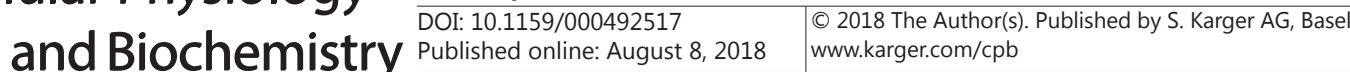

\section{RNA pull-down}

SMMC-7721 cells were transfected with biotinylated miRNA, and were collected at $48 \mathrm{~h}$ after transfection. The cell lysates were incubated with M-280 streptavidin magnetic beads (Invitrogen, San Diego, CA, USA). The bound RNAs were purified using TRIzol reagent (Invitrogen) for further qRT-PCR analysis.

\section{Statistical analysis}

SPSS 17.0 software was used for all statistical analyses. The significance of the differences between two groups was estimated with the Student's $t$-test. Multiple group comparisons were made and analyzed with one-way analysis of variance. The overall survival probability was analyzed and evaluated by Kaplan-Meier analyses and the log-rank test. Cox proportional hazards model was used to identify factors associated with the overall survival rate of HCC patients through multivariate analysis. The statistically significant correlation between TUG1 and miR-142-3p as well as that between miR-142-3p and ZEB1 in 92 cases of HCC tissues was analyzed with Spearman's correlation analysis. $\mathrm{P}$ values less than 0.05 were considered statistically significant.

\section{Results}

Downregulation of miR-142-3p is closely correlated with poor prognosis of HCC patients

To explore the biological role of miR-142-3p in HCC, the levels of miR-142-3p in 92 HCC tissues were measured by quantitative real-time PCR (qRT-PCR). As illustrated in Fig. 1A, the expression level of miR142-3p was significantly lower in HCC tissues than in corresponding normal tissues $(\mathrm{P}<0.001)$. Next, we analyzed the correlation between miR-142-3p and the clinical pathological parameters of HCC patients. According to the mean level of miR-142-3p, 92 HCC patient samples were divided into two groups: miR142-3p high-expression group $(n=45)$ and miR-142-3p
Table 1. Correlation between miR-142$3 p$ expression and clinical Features. ( $\mathrm{n}=92)$. Low/high by the sample mean. Pearson $\chi^{2}$ test. ${ }^{*} \mathrm{P}<0.05,{ }^{* *} \mathrm{P}<0.01$ was considered statistically significant

\begin{tabular}{|c|c|c|c|}
\hline \multirow{2}{*}{ Variable } & \multicolumn{2}{|c|}{ miR-142-3p expression } & \multirow{2}{*}{ P-value } \\
\hline & low & high & \\
\hline \multicolumn{4}{|l|}{ Age } \\
\hline$<60$ & 35 & 41 & \multirow[t]{2}{*}{0.278} \\
\hline$\geq 60$ & 10 & 6 & \\
\hline \multicolumn{4}{|l|}{ Sex } \\
\hline Male & 26 & 18 & \multirow[t]{2}{*}{0.094} \\
\hline Female & 19 & 29 & \\
\hline \multicolumn{4}{|l|}{ HbsAg } \\
\hline Negative & 17 & 20 & \multirow[t]{2}{*}{0.675} \\
\hline Positive & 28 & 27 & \\
\hline \multicolumn{4}{|l|}{ ALT } \\
\hline$\leq 45$ & 31 & 39 & \multirow[t]{2}{*}{0.145} \\
\hline$>45$ & 14 & 8 & \\
\hline \multicolumn{4}{|l|}{ AFP } \\
\hline$\leq 13.6$ & 34 & 33 & \multirow[t]{2}{*}{0.642} \\
\hline$>13.6$ & 11 & 14 & \\
\hline \multicolumn{4}{|c|}{ Vascular invasion } \\
\hline Absent & 13 & 25 & \multirow[t]{2}{*}{$0.021^{*}$} \\
\hline Present & 32 & 22 & \\
\hline \multicolumn{4}{|l|}{ Cirrhosis } \\
\hline Absent & 20 & 39 & \multirow[t]{2}{*}{$<0.001^{* *}$} \\
\hline Present & 25 & 8 & \\
\hline \multicolumn{4}{|c|}{ Tumor size } \\
\hline$\leq 5$ & 17 & 36 & \multirow[t]{2}{*}{$<0.001^{* *}$} \\
\hline$>5$ & 28 & 11 & \\
\hline \multicolumn{4}{|c|}{ Edmondson grade } \\
\hline I-II & 20 & 34 & \multirow[b]{2}{*}{$0.011^{*}$} \\
\hline III-IV & 25 & 13 & \\
\hline
\end{tabular}

Fig. 1. Downregulation of miR142-3p was closely correlated with poor prognosis in HCC patients. A. The level of miR-142-3p in HCC tissues was measured by qRT-PCR. B. The association between miR142-3p and overall survival rate of HCC patients was analyzed by Kaplan-Meier analysis (log-rank

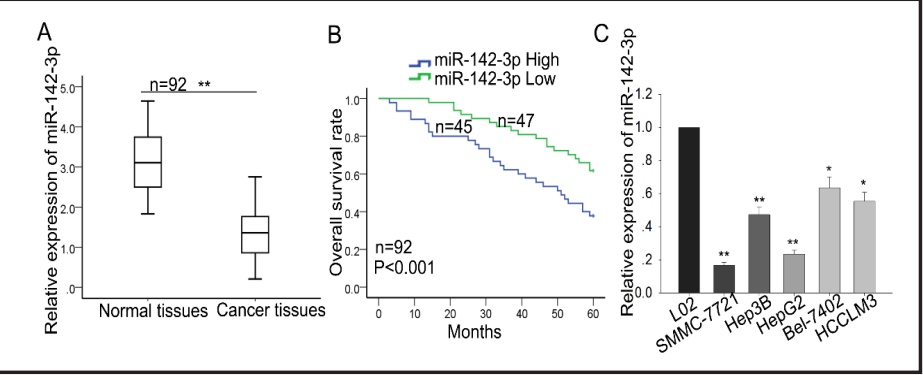
test). C. The level of miR-142-3p in HCC cell lines was measured by qRT-PCR. Data are represented as the mean \pm standard deviation (SD) of three independent experiments. The $\mathrm{p}$-value represents the comparison between groups $\left({ }^{*} \mathrm{p}<0.05,{ }^{* *} \mathrm{p}<0.01\right)$. 
low-expression group $(\mathrm{n}=47)$. As shown in Table 1, low miR-142-3p levels were significantly correlated with vascular invasion $(\mathrm{P}=0.021)$, cirrhosis $(\mathrm{P}<0.001)$, tumor size $(\mathrm{P}<0.001)$, and Edmondson grade $(\mathrm{P}=0.011)$, but not with age, sex, hepatitis $B$ surface antigen, alanine aminotransferase, and alpha fetoprotein levels $(\mathrm{P}>0.05)$. Moreover, Kaplan-Meier analysis (log-rank test) revealed that a low level of miR-142-3p was associated with poor prognosis $(\mathrm{P}<0.001$, log-rank test; Fig. 1B). Additionally, Cox regression analysis showed that a low level of miR-142-3p could be an independent prognostic factor $(\mathrm{P}<0.001)$, as well as a predictor of vascular invasion and Edmondson grade (Table 2). We evaluated the level of miR-142-3p in a panel of HCC cell lines (SMMC7721, Hep3B, HepG2, Bel7402, HCCLM3), and found that miR-142-3p was notably downregulated in HCC cells compared with the L02 human normal liver epithelial cell line; the lowest miR-142-3p expression levels were found in SMMC-7721 and HepG2 cells (Fig. 1C). Collectively, these findings indicate that miR-142$3 p$ acts as a tumor suppressor in HCC progression and might be a promising prognostic marker for HCC patients.

miR-142-3p suppresses the proliferation, migration, and invasion of HCC cells

According to abovementioned findings, we postulated that miR-142-3p might be associated with tumor metastasis. Because the lowest
Table 2. Multivariate and univariate analyses of prognostic parameters in patients with HCC by Cox regression analysis. Cox regression analysis showed the positive, independent prognostic importance of miR142-3p expression $\left(\mathrm{P}=0.013^{*}\right) .{ }^{*} \mathrm{P}<0.05$ and ${ }^{* *} \mathrm{P}<0.01$ were considered statistically significant

\begin{tabular}{|c|c|c|c|}
\hline \multirow[b]{2}{*}{ Variable } & \multirow{2}{*}{$\begin{array}{l}\text { Univariate analysis } \\
\text { Category }\end{array}$} & \multicolumn{2}{|c|}{ Multivariate analysis } \\
\hline & & P-value & P-value \\
\hline \multirow[t]{2}{*}{ Age } & $<60$ & 0.307 & \\
\hline & $\geq 60$ & & \\
\hline \multirow[t]{2}{*}{ Sex } & Male & 0.364 & \\
\hline & Female & & \\
\hline HbsAg & Negative & 0.352 & \\
\hline ALT & $\leq 45$ & 0.688 & \\
\hline \multirow[t]{2}{*}{ AFP } & $\leq 13.6$ & 0.267 & \\
\hline & $>13.6$ & & \\
\hline \multirow[t]{2}{*}{ Vascular invasion } & Absent & $0.014^{*}$ & $0.029^{*}$ \\
\hline & Present & & \\
\hline \multirow[t]{2}{*}{ Cirrhosis } & Absent & 0.245 & \\
\hline & Present & & \\
\hline \multirow[t]{2}{*}{ Tumor size } & $\leq 5$ & 0.318 & \\
\hline & $>5$ & & \\
\hline \multirow[t]{2}{*}{ Edmondson grade } & I-II & $0.015^{*}$ & $0.030^{*}$ \\
\hline & III-IV & & \\
\hline \multirow[t]{2}{*}{ miR-142-3p expression } & Low & & 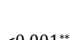 \\
\hline & High & & \\
\hline
\end{tabular}

Fig. 2. miR-142-3p suppressed the proliferation, migration, and invasion of HCC cell. A. SMMC-7721 and HepG2 cells were transfected with miR-142-3p mimics, and transfection efficiency was determined with RT-qPCR. B-C. Wound healing and transwell assays were performed to measure the effect of miR$142-3 p$ on the migration and invasion of HCC cells. D-E. The effect of miR-142-3p on cell proliferative ability was evaluated by MTT and colony formation assays. Data are represented as the mean \pm SD of three independent experiments. The p-value represents the comparison between groups $\left({ }^{*} \mathrm{p}<0.05,{ }^{* *} \mathrm{p}<0.01\right)$.

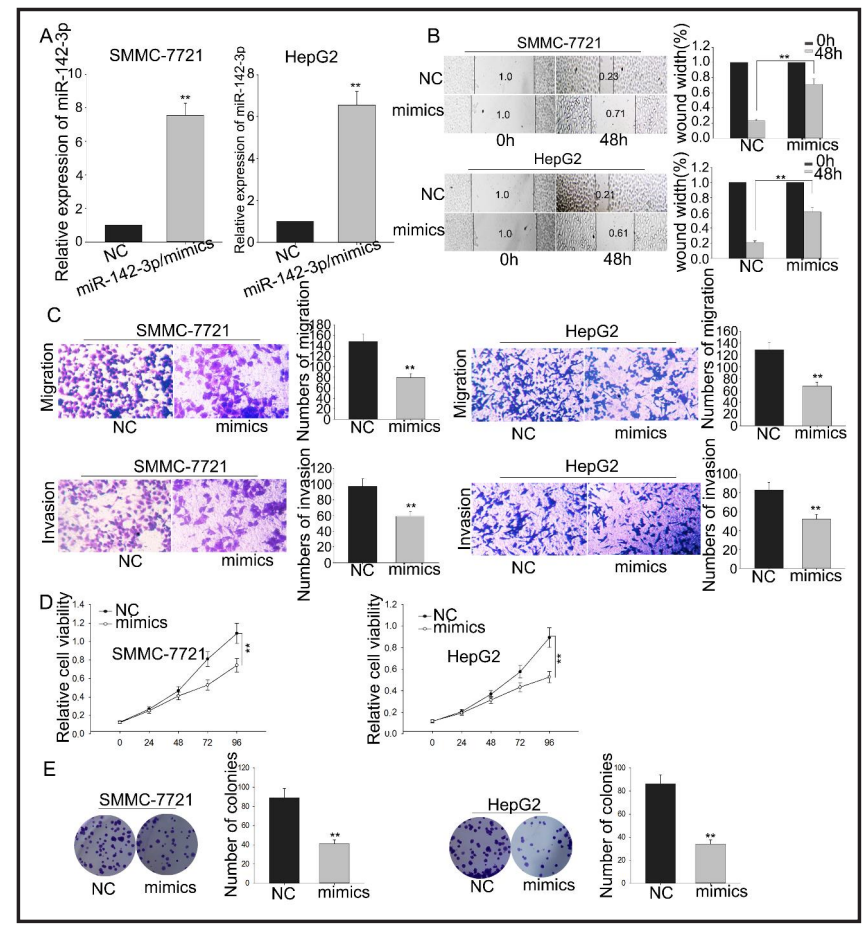


Fig. 3. Ectopic expression of miR-142-3p reversed the phenotype from EMT to MET in HCC cells. A-B. Western blot analysis was utilized to determine the effect of miR142-3p on the expression levels of EMT markers. C-D. Immunofluorescence was performed to assess the levels of EMT markers in HCC cells. Data are represented as the mean \pm $\mathrm{SD}$ of three independent experiments. The $\mathrm{p}$-value represents the comparison between groups $\left({ }^{*} \mathrm{p}<0.05,{ }^{* *} \mathrm{p}<0.01\right)$.

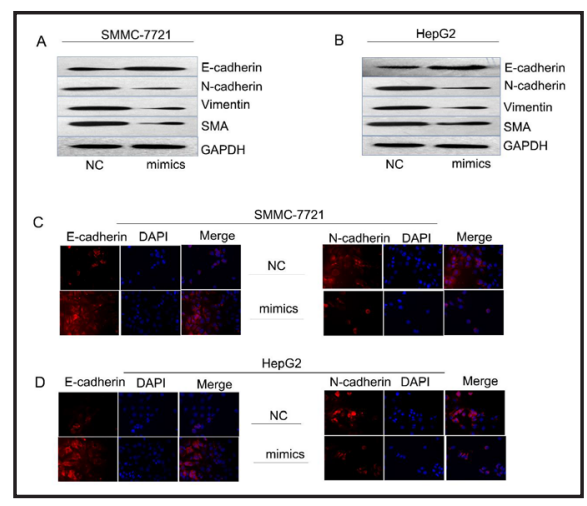

Fig. 4. ZEB1 was identified as a target of miR-142-3p. A. The binding sites between miR-142-3p and ZEB1 was predicted. B. The level of ZEB1 in HCC cells was measured. C. The effect of miR-142-3p on the mRNA and protein levels of ZEB1 was analyzed and investigated by qRT-PCR and western blot analysis. D. Luciferase reporter assays were applied to detect the interaction between miR-142-3p and ZEB1. E. The level of ZEB1 in HCC tissues and corresponding normal tissues was detected by qRT-PCR. F. The correlation between miR-142-3p and ZEB1 was analyzed with Spearman's correlation analysis $(\mathrm{r}=-0.653, \mathrm{P}<0.001)$. Data are represented as the mean $\pm \mathrm{SD}$ of three independent experiments. The $\mathrm{p}$-value represents the comparison between groups ( $\left.\mathrm{p} p<0.05,{ }^{* *} \mathrm{p}<0.01\right)$.

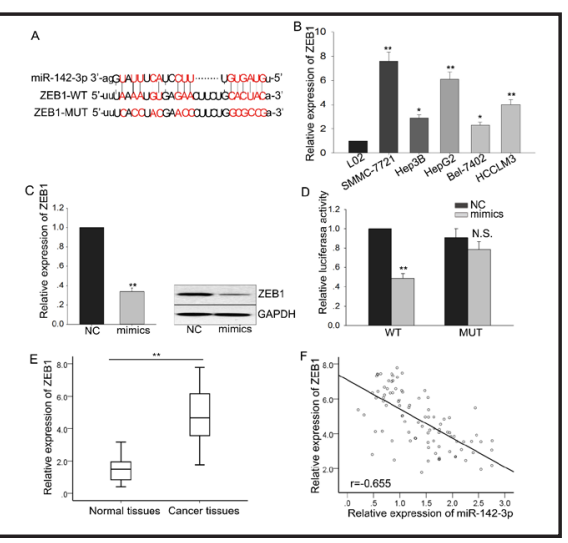

miR-142-3p expression was found in SMMC-7721 and HepG2 cells, they were chosen for subsequent experiments. To assess the biological effects of miR-142-3p on cell migration and invasion, we transfected SMMC-7721 and HepG2 cells with miR-142-3p mimics; the transfection efficiency was determined by RT-qPCR (Fig. 2A). Wound healing and transwell assays revealed that miR-142-3p mimics suppressed the migratory and invasive capacity of SMMC-7721 and HepG2 cells (Fig. 2B, C). Upregulation of miR-142-3p weakened the proliferative ability of HCC cells (Fig. 2D, E). These results showed that miR-142-3p inhibited the metastasis of HCC by regulating cell migration and invasion.

Ectopic expression ofmiR-142-3p reverses the phenotype from the EMT to the mesenchymalepithelial transition in HCC cells

The EMT process is crucial for malignant transformation; thus, we evaluated the effects of miR-142-3p on the EMT process. Western blot analysis showed that after transfection with miR-142-3p mimics, levels of the epithelial marker (E-cadherin) in HCC cells were significantly increased, whereas those of the mesenchymal markers $\mathrm{N}$-cadherin, vimentin, and $\alpha$-smooth muscle actin ( $\alpha$-SMA) were decreased (Fig. 3A, B). Consistent with these data, immunofluorescence experiments confirmed the effect of miR-142-3p on EMT progress of HCC cells (Fig. 3C, D). These results indicated that miR-142-3p restrained acquisition of the EMT phenotype in HCC cells, which helped suppress the metastatic ability of HCC cells.

\section{ZEB1 is a target of miR-142-3p}

To explore the downstream molecular mechanism of miR-142-3p in HCC cells, we utilized three publicly available databases (TargetScan, starBase, and miRanda). ZEB1, a key regulator of the EMT progress, was found to be a target of miR-142-3p (Fig. 4A). High ZEB1 levels were found in HCC cell lines (SMMC7721, Hep3B, HepG2, Bel-7402, HCCLM3) compared with L02 cells (Fig. 4B). We measured the effects of miR-142-3p on the mRNA and protein levels of ZEB1, and found that miR-142-3p overexpression decreased these levels (Fig. 4C). 
Fig. 5. Overexpression of ZEB1 reversed the effects of miR-142-3p in HCC cells. A-C. Overexpressed ZEB1 partially abrogated the inhibitory effects of miR-142-3p on the cell metastatic abilities and EMT progress. Data are represented as the mean \pm SD of three independent experiments. The p-value represents the comparison between groups $\left({ }^{*} \mathrm{p}<0.05,{ }^{* *} \mathrm{p}<0.01\right)$.

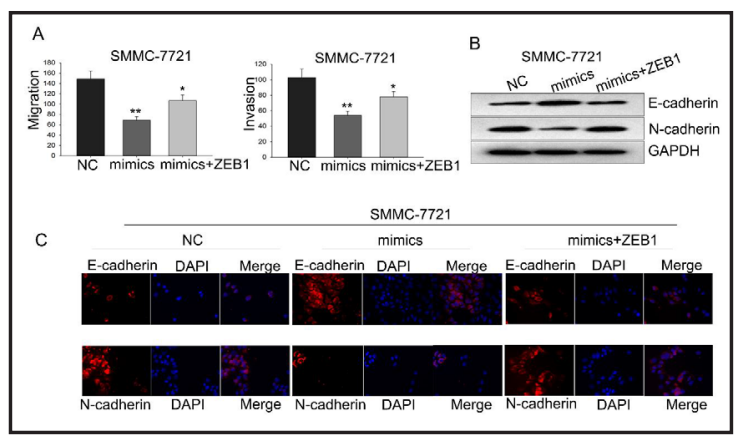

Fig. 6. TUG1 acted as an oncogene and negatively regulated miR-1423p. A. The level of TUG1 in HCC tissues and corresponding normal tissues was detected by qRT-PCR. B. SMMC-7721 and HepG2 cells were transfected with si-TUG1 or siRNA to silence TUG1. C-D. MTT and colony formation assays were conducted to detect the effect of TUG1 on the proliferative ability of HCC cells. E. Transwell assays were performed to evaluate the effect of TUG1 on cell migration and invasion. F. The effect of TUG1 on miR-142-3p expression was detected by qRT-PCR. G. The correlation between miR-142$3 p$ and TUG1 was analyzed by Spearman's correlation analysis ( $\mathrm{r}=-0.741, \quad \mathrm{P}<0.001)$. Data are represented as the mean \pm SD of three independent experiments. The p-value represents the comparison between groups $\left({ }^{*} \mathrm{p}<0.05,{ }^{* *} \mathrm{p}<0.01\right)$.

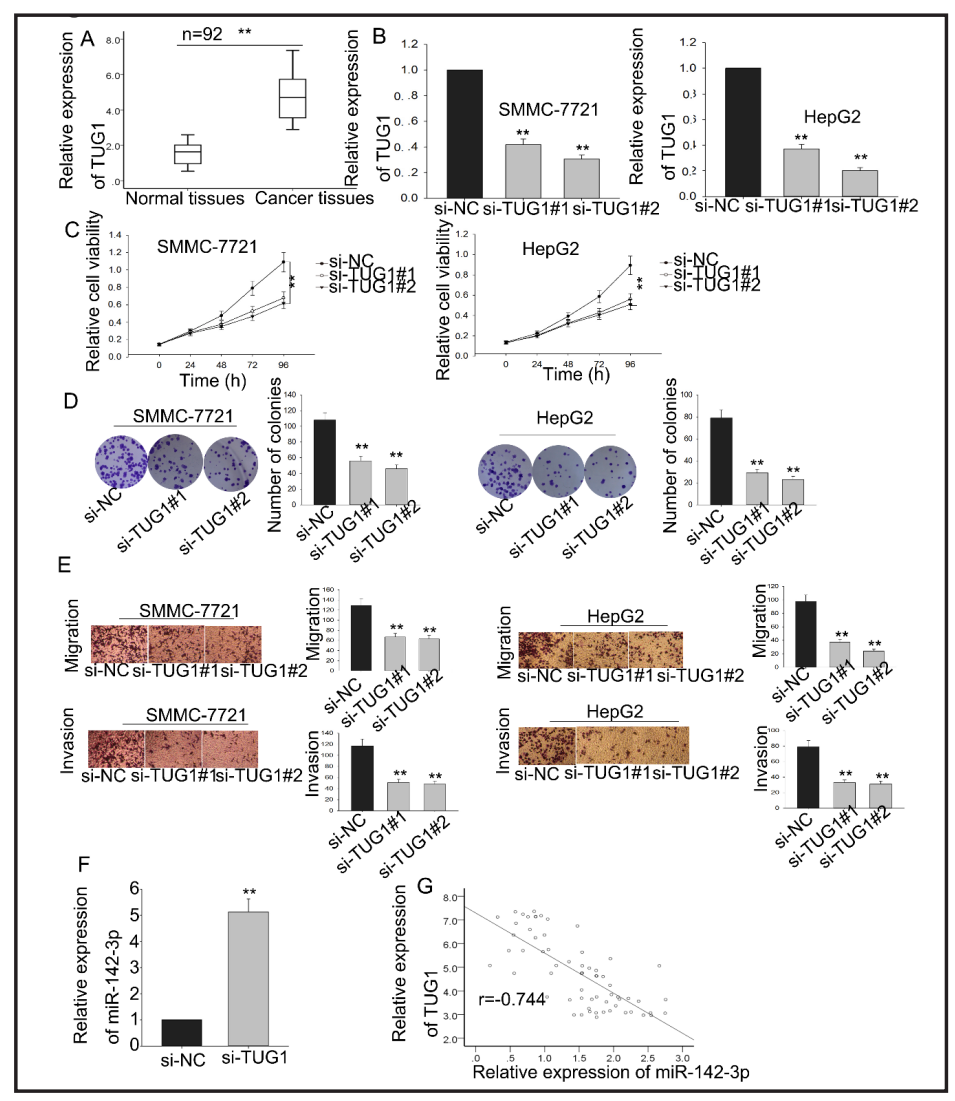

Luciferase reporter assays revealed that miR-142-3p overexpression notably reduced the luciferase activity of the wild-type 3' untranslated region (WT 3'-UTR) of ZEB1 but not that of the mutant (MUT) 3'-UTR of ZEB1 (Fig. 4D). Furthermore, qRT-PCR experiments showed that ZEB1 was upregulated in HCC tissues (Fig. 4E), and Spearman's correlation analysis showed that miR-142-3p expression was inversely correlated with that of ZEB1 ( $\mathrm{r}=-0.653$, $\mathrm{P}<0.001$, Fig. 4F). Therefore, we conclude that ZEB1 is a direct target of miR-142-3p in HCC.

Overexpression of ZEB1 reverses the effects of miR-142-3p in HCC cells

Rescue assays were performed to further confirm the impact of ZEB1 on miR-142-3pmediated function in HCC cells. As expected, ZEB1 overexpression partially abrogated the inhibitory effects of miR-142-3p on the migration and invasion of SMMC7721 cells (Fig. 5A). Additionally, overexpressed ZEB1 partially reversed the MET to EMT in SMMC7721 cells (Fig. 5B, C). These findings suggest that ZEB1 is a target of miR-142-3p and mediates the function of miR-142-3p in HCC. 
TUG1 acts as an oncogene and negatively regulates miR-142-3p

To determine the function of TUG1, we measured its level in HCC tissues and corresponding normal tissues, and found that TUG1 was upregulated in HCC tissues (Fig. 6A). Subsequently, TUG1 was knocked down by transfection with specific small interfering RNAs (siRNAs); the transfection efficiency was determined by qRT-PCR (Fig. 6B). Knockdown of TUG1 repressed the proliferation, migration, and invasion of HCC cells compared with the negative controls (Fig. 6C-E). The functions of TUG1 in HCC cells were different from those of miR-142-3p. Accordingly, we hypothesized that TUG1 is inversely correlated with miR-142$3 p$ in HCC. To test our hypothesis, qRT-PCR was conducted to determine the effect of TUG1 on the expression level of miR-142-3p. Silenced TUG1 clearly increased the level of miR142-3p (Fig. 6F), and Spearman's correlation analysis confirmed the inverse relationship between TUG1 and miR-142-3p (Fig. 6G). Taken together, we conclude that IncRNA TUG1 plays an oncogenic role in HCC and is inversely correlated with miR-142-3p.

Fig. 7. TUG1 regulated ZEB1 by competitively binding with miR-1423p. A. The binding sites for miR-142$3 p$ harboring the TUG1 are presented. B. Ago2-RIP assays were applied to verify binding between TUG1 and miR$142-3 p$. IgG was used as the negative control, and SNRNP70 binding to U1 was used as the positive control. C. RNA pulldown assay was used to confirm the combination between miR-142-3p and TUG1. D. Luciferase reporter assay was conducted to verify the interaction between TUG1 and miR-142-3p. E. qRT-PCR was performed to measure the effect of silenced TUG1 on the level of ZEB1. Immunofluorescence and western blot assays were performed to measure the effect of silenced TUG1 on EMT acquisition. F. The correlation between TUG1 and ZEB1 in HCC tissues was analyzed. Data are represented as

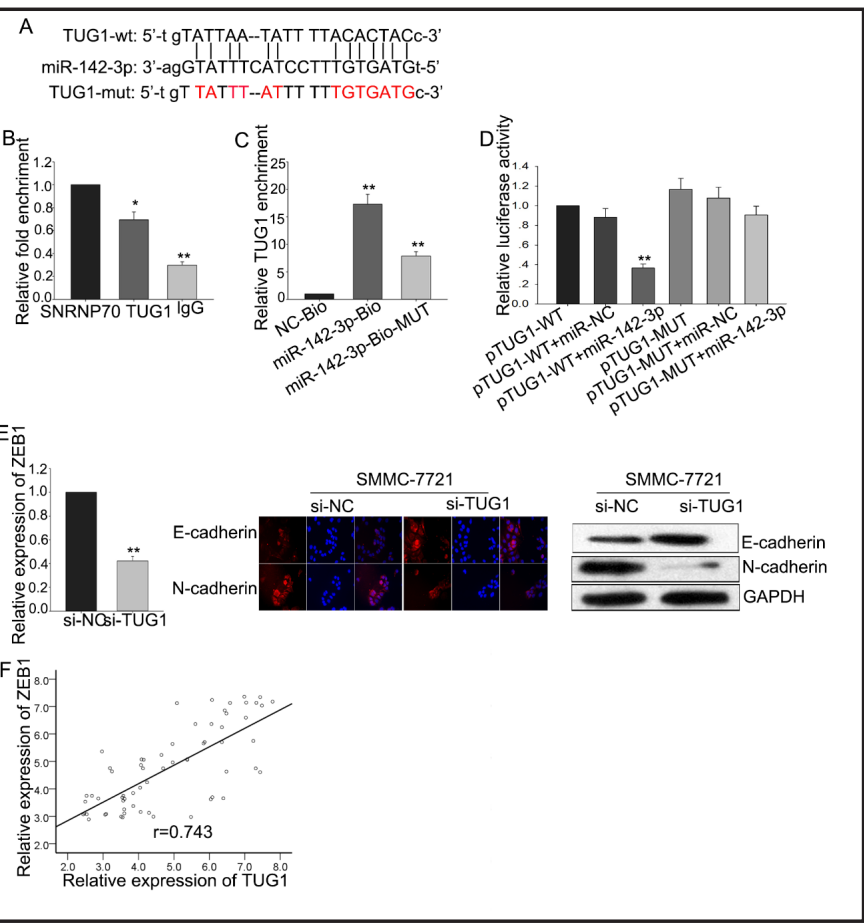
the mean \pm SD of three independent experiments. The $\mathrm{p}$-value represents the comparison between groups $\left({ }^{*} \mathrm{p}<0.05,{ }^{* *} \mathrm{p}<0.01\right)$.

Fig. 8. TUG1/miR-142-3p/ZEB1 axis contributed to the proliferation and EMT in vivo. A. Growth curve of tumor volumes and representative tumor photographs were generated and obtained after 28 days. B. Images of Ki-67 immunostaining were obtained from the transplanted tumors. C. The levels of miR-142-3p and ZEB1 in tumor tissues were detected by qRT-PCR. D. Western blotting was utilized to detect the expression level of E-cadherin and $\mathrm{N}$-cadherin. Data are represented as the mean \pm $\mathrm{SD}$ of three independent experiments. The p-value represents the comparison between groups $\left({ }^{*} \mathrm{p}<0.05\right.$, ${ }^{* *} \mathrm{p}<0.01$ ).

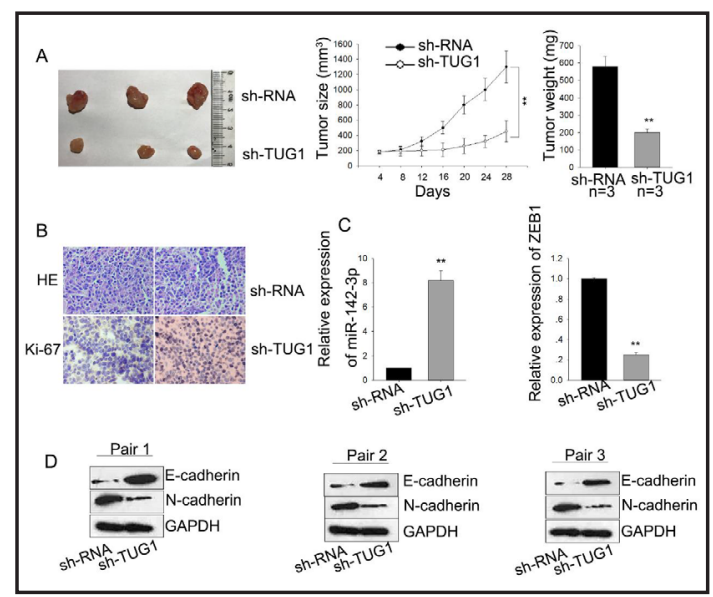




\section{Cellular Physiology Cell Physiol Biochem 2018;48:1928-1941 \begin{tabular}{l|l} 
and Biochemistry Published online: August 8, 2018 & $\begin{array}{l}\text { DO } 2018 \text { The Author(s). Published by S. Karger AG, Basel } \\
\text { www.karger.com/cpb }\end{array}$
\end{tabular}}

He et al.: The Function of TUG1-miR-142-3p-ZEB1 Axis in HCC

TUG1 regulates ZEB1 by competitively binding with miR-142-3p

In recent years, the patterns of ceRNA interactions have been widely reported. In a ceRNA model, lncRNA release or upregulate target mRNA of miRNAs by competitively binding with the same miRNAs Zhang et al. [27-31]. TUG1 participates in the progression and development of multiple tumors by acting as a ceRNA Zhang et al. [22, 24, 26, 32-36]. To determine whether TUG1 acts as a ceRNA in HCC, we applied bioinformatics analysis (http://starbase.sysu.edu. cn/browseNcRNA.php) to identify the binding site between TUG1 and miR-142-3p (Fig. 7A). Thereafter, we performed a series of experiments (RNA immunoprecipitation [RIP], RNA pull-down, and luciferase reporter assay) to verify the binding relationship between TUG1 and miR-142-3p. As illustrated in Fig. 7B, the fold enrichment of TUG1 in the RIP experiments was markedly higher in the beads harboring argonaute-2 (Ago2). IgG was used as the negative control, and small nuclear ribonucleoprotein U1 subunit 70 (SNRNP70) binding with U1 was used as the positive control. In the RNA pull-down assay, biotinylated miR-142-3p captured more TUG1 than did biotinylated miR-142-3p-mut (Fig. 7C). The luciferase reporter assay showed that overexpression of miR-142-3p markedly decreased the luciferase activity of wild-type TUG1 but not mutant TUG1 (Fig. 7D). These findings confirmed the interaction between TUG1 and miR-142-3p. Additionally, we assessed the effects of TUG1 on ZEB1 and EMT-related proteins. Knockdown of TUG1 inhibited the expression of ZEB1 and EMT progression in SMMC-7721 cells (Fig. 7E). Finally, the positive correlation between TUG1 and ZEB1 was verified by Spearman's correlation analysis (Fig. 7F). Taken together, these data showed that TUG1 facilitated ZEB1-mediated EMT acquisition in HCC cells by acting as a ceRNA through competitively sponging miR-142-3p.

TUG1/miR-142-3p/ZEB1 axis contributes to cell proliferation and the EMT acquisition in vivo

To further confirm that the TUG1/miR-142/ZEB1 axis affects the tumorigenesis of HCC in vivo, SMMC-7721 cells stably transfected with single hairpin-TUG1 (sh-TUG1) or control vector were inoculated into nude mice. Tumors were substantially smaller in the sh-TUG1 group than in the control group; tumor volume and weight were also significantly reduced by silenced TUG1 (Fig. 8A). Tumors formed from stably sh-TUG1-transfected SMMC-7721 cells exhibited weakened positivity for Ki-67 compared with control cells (Fig. 8B). qRTPCR assays revealed that the expression level of miR-142-3p was increased in tumor tissues derived from TUG1-downregulated SMMC-7721 cells (Fig. 8C), whereas the expression level of ZEB1 was decreased in tumor tissues derived from TUG1-downregulated SMMC-7721 cells (Fig. 8C). Moreover, western blot analysis revealed that silenced TUG1 significantly reduced the level of $\mathrm{N}$-cadherin but increased the level of E-cadherin (Fig. 8D). These in vivo data further demonstrated the oncogenic function of the TUG1/miR-142-3p/ZEB1 axis.

\section{Discussion}

HCC, a highly vascularized tumor, is one of the most prevalent fatal malignancies worldwide, and its increasing incidence has become a major health problem. HCC is often accompanied by frequent metastasis (intrahepatic or extrahepatic), and limited therapeutic approaches have resulted in a high mortality rate. Therefore, it is an urgent need to explore the underlying molecular mechanisms of HCC metastasis.

Integrative genomic studies have shown that $\sim 98 \%$ of the human genome transcripts are ncRNAs. Based on length, these ncRNAs can be divided into two groups: short ncRNAs ( $<200$ nucleotides) and long ncRNAs ( $>200$ nucleotides). As a type of typical short ncRNAs, miRNAs have been shown to be dysregulated in many cancer types. Additionally, miRNAs can participate in tumorigenesis by regulating target genes via translational suppression or mRNA degradation Bash-Imam et al. [37-42]. For example, Tang et al. [43]. reported that dysregulated miR-218 is associated with the clinicopathological characteristics of cervical 
cancer patients. Wu et al. showed that miR-655-3p functioned as a tumor suppressor in HCC by regulating a disintegrin and metalloproteinase domain-containing protein 10 and betacatenin pathway Wu et al. [44].

In this study, we demonstrated that miR-142-3p was downregulated in HCC tissues and cell lines. Furthermore, our data showed that low levels of miR-142-3p were positively associated with vascular invasion $(\mathrm{P}=0.021)$, cirrhosis $(\mathrm{P}=0.000)$, tumor size $(\mathrm{P}=0.000)$, and Edmondson grade $(\mathrm{P}=0.011)$ and predicted poor prognosis in HCC patients. Because high metastasis ability is a main feature of HCC, we focused on the effects of miR-142-3p on metastasis and the EMT phenotype. The cellular experiments illustrated that miR-142-3p overexpression significantly repressed cell metastasis and reversed the EMT. EMT events have been widely found in the metastasis process of various cancers Ruan et al. [45-47]. ZEB1 is a critical regulator of the EMT Zhang et al. $[48,49]$. In the current study, we found that miR142-3p expression was inversely correlated with ZEB1 expression in HCC cells and tissues. The results of the luciferase reporter assays revealed that ZEB1 is a direct downstream target of miR-142-3p in HCC. Furthermore, rescue assays showed that overexpressed ZEB1 abolished the effects of miR-142-3p on the migration, invasion, and EMT process. Therefore, miR-142-3p exerts its function in HCC by targeting ZEB1.

Endogenous transcripts containing miRNA response elements (MREs) can interact with or co-regulate each other by acting as endogenous miRNA sponges or ceRNAs, thereby forming large-scale regulatory network across the transcriptome Salmena et al. [31, 50]. Numerous studies have documented that IncRNAs harbor potential MREs and function as competitive platforms for miRNAs. For instance, Ye et al. [51]. demonstrated that the lncRNA GAS5 suppresses cell growth and the EMT in osteosarcoma by regulating the miR-221/ ARHI pathway. Huang et al. [52]. reported that the novel long intergenic noncoding RNA upregulated in CRC (UCC) promoted CRC progression by sponging miR-143. TUG1 plays a role in the regulation of many cancer types by functioning as a ceRNA Wang et al. [22, 32, $34,35,53$. Based on the results of our bioinformatics analysis, it was hypothesized that miR-142-3p could be negatively regulated by TUG1. Our findings revealed that the level of TUG1 in HCC tissues was inversely correlated with that of miR-142-3p. In addition, TUG1 negatively regulated the expression level of miR-142-3p. RIP, RNA pull-down, and luciferase reporter assays were further performed to confirm the interaction between miR-142-3p and TUG. Finally, silenced TUG1 significantly reduced the levels of ZEB1 and EMT-related proteins.

The results of this study showed that miR-142-3p acted as a tumor suppressor in HCC, and its downregulation was associated with poor prognosis in HCC patients. Cellular and mechanistic assays revealed that the TUG1-miR-142-3p-ZEB1/EMT axis contributes to the metastasis of HCC cells and might be a therapeutic target for this disease.

\section{Acknowledgements}

The authors thank all members involved in this study.

This work was supported by the National Natural Science Foundation of China (Grant Nos. 81374014 and 81672430), the Zhejiang Provincial Science and Technology Projects (Grant Nos. 2015C33264, 2017C33212, and 2017C33213), and the Zhejiang Provincial Medical and Healthy Science and Technology Projects (Grant Nos. 2013KYA228 and 2016KYA180).

\section{Disclosure Statement}

No conflict of interests exists. 


\section{Cellular Physiology Cell Physiol Biochem 2018;48:1928-1941 \begin{tabular}{l|l} 
and Biochemistry Published online: August 8, 2018 & $\begin{array}{l}\text { C) 2018 The Author(s). Published by S. Karger AG, Basel } \\
\text { www.karger.com/cpb }\end{array}$ \\
\hline
\end{tabular}}

He et al.: The Function of TUG1-miR-142-3p-ZEB1 Axis in HCC

\section{References}

1 Siegel RL, Miller KD, Jemal A: Cancer statistics, 2016. CA Cancer J Clin 2016;66:7-30.

-2 Chen W, Zheng R, Baade PD, Zhang S, Zeng H, Bray F, Jemal A, Yu XQ He J: Cancer statistics in China, 2015. CA Cancer J Clin 2016;66:115-132.

3 Xiong H, Ni Z, He J, Jiang S, Li X, He J, Gong W, Zheng L, Chen S, Li B, Zhang N, Lyu X, Huang G, Chen B, Zhang Y, He F: LncRNA HULC triggers autophagy via stabilizing Sirt1 and attenuates the chemosensitivity of HCC cells. Oncogene 2017;36:3528-3540.

- Wang Y, Hu Y, Wu G, Yang Y, Tang Y, Zhang W, Wang K, Liu Y, Wang X, Li T: Long noncoding RNA PCAT-14 induces proliferation and invasion by hepatocellular carcinoma cells by inducing methylation of miR-372. Oncotarget 2017;8:34429-34441.

5 Tang J, Xie Y, Xu X, Yin Y, Jiang R, Deng L, Tan Z, Gangarapu V, Tang J, Sun B: Bidirectional transcription of Linc00441 and RB1 via H3K27 modification-dependent way promotes hepatocellular carcinoma. Cell death Dis 2017;8:e2675.

6 Ni W, Zhang Y, Zhan Z, Ye F, Liang Y, Huang J, Chen K, Chen L, Ding Y: A novel lncRNA uc.134 represses hepatocellular carcinoma progression by inhibiting CUL4A-mediated ubiquitination of LATS1.J Hematol Oncol 2017;10:91.

7 Li C, Miao R, Liu S, Wan Y, Zhang S, Deng Y, Bi J, Qu K, Zhang J, Liu C: Down-regulation of miR-146b-5p by long noncoding RNA MALAT1 in hepatocellular carcinoma promotes cancer growth and metastasis. Oncotarget 2017;8:28683-28695.

8 Mattick JS: The genetic signatures of noncoding RNAs. PLoS Genet 2009;5:e1000459.

-9 Keller C, Kulasegaran-Shylini R, Shimada Y, Hotz HR, Buhler M: Noncoding RNAs prevent spreading of a repressive histone mark. Nat Struct Mol Biol 2013;20:1340.

10 Lv J, Liu H, Huang Z, Su J, He H, Xiu Y, Zhang Y, Wu Q: Long non-coding RNA identification over mouse brain development by integrative modeling of chromatin and genomic features. Nucleic Acids Res 2013;41:10044-10061.

11 Ohtsuka M, Ling H, Doki Y, Mori M, Calin GA: MicroRNA Processing and Human Cancer. J Clin Med 2015;4:1651-1667.

12 Baer C, Claus R, Plass C: Genome-wide epigenetic regulation of miRNAs in cancer. Cancer Res 2013;73:473477.

13 Bartel DP: MicroRNAs: genomics, biogenesis, mechanism, and function. Cell 2004;116:281-297.

-14 Chen Y, Zhou X, Qiao J, Bao A: MiR-142-3p Overexpression Increases Chemo-Sensitivity of NSCLC by Inhibiting HMGB1-Mediated Autophagy. Cell Physiol Biochem 2017;41:1370-1382.

15 Liu K, Huang J, Ni J, Song D, Ding M, Wang J, Huang X, Li W: MALAT1 promotes osteosarcoma development by regulation of HMGB1 via miR-142-3p and miR-129-5p. Cell cycle 2017;16:578-587.

16 Xue Y, Ni T, Jiang Y, Li Y: Long Noncoding RNA GAS5 Inhibits Tumorigenesis and Enhances Radiosensitivity by Suppressing miR-135b Expression in Non-Small Cell Lung Cancer. Oncol Res 2017;25:1305-1316.

17 Wang W, Zhuang Q, Ji K, Wen B, Lin P, Zhao Y, Li W, Yan C: Identification of miRNA, IncRNA and mRNAassociated ceRNA networks and potential biomarker for MELAS with mitochondrial DNA A3243G mutation. Sci Rep 2017;7:41639.

-18 Yang S, Ning Q Zhang G, Sun H, Wang Z, Li Y: Construction of differential mRNA-lncRNA crosstalk networks based on ceRNA hypothesis uncover key roles of lncRNAs implicated in esophageal squamous cell carcinoma. Oncotarget 2016;7:85728-85740.

19 Wang H, Niu L, Jiang S, Zhai J, Wang P, Kong F, Jin X: Comprehensive analysis of aberrantly expressed profiles of IncRNAs and miRNAs with associated ceRNA network in muscle-invasive bladder cancer. Oncotarget 2016; 7:86174-86185.

20 Ma MZ, Chu BF, Zhang Y, Weng MZ, Qin YY, Gong W, Quan ZW: Long non-coding RNA CCAT1 promotes gallbladder cancer development via negative modulation of miRNA-218-5p. Cell Death Dis 2015;6:e1583.

21 Wang WT, Ye H, Wei PP, Han BW, He B, Chen ZH, Chen YQ: LncRNAs H19 and HULC, activated by oxidative stress, promote cell migration and invasion in cholangiocarcinoma through a ceRNA manner. J Hematol Oncol 2016;9:117.

22 Wang Y, Yang T, Zhang Z, Lu M, Zhao W, Zeng X, Zhang W: Long non-coding RNA TUG1 promotes migration and invasion by acting as a ceRNA of miR-335-5p in osteosarcoma cells. Cancer Sci 2017;108:859-867. 


\section{Cellular Physiology Cell Physiol Biochem 2018;48:1928-1941 \begin{tabular}{l|l} 
and Biochemistry Published 1159/000492517 & $\begin{array}{l}\text { C) } 2018 \text { The Author(s). Published by S. Karger AG, Basel } \\
\text { www.karger.com/cpb }\end{array}$ \\
\hline
\end{tabular} \\ He et al.: The Function of TUG1-miR-142-3p-ZEB1 Axis in HCC}

23 Ma HW, Xie M, Sun M, Chen TY, Jin RR, Ma TS, Chen QN, Zhang EB, He XZ, De W, Zhang ZH: The pseudogene derived long noncoding RNA DUXAP8 promotes gastric cancer cell proliferation and migration via epigenetically silencing PLEKHO1 expression. Oncotarget 2017;8:52211-52224.

24 Ren K, Li Z, Li Y, Zhang W, Han X: Long Noncoding RNA Taurine-Upregulated Gene 1 Promotes Cell Proliferation and Invasion in Gastric Cancer via Negatively Modulating miRNA-145-5p. Oncol Res 2017;25:789-798.

-25 Zhao L, Sun H, Kong H, Chen Z, Chen B, Zhou M: The Lncrna-TUG1/EZH2 Axis Promotes Pancreatic Cancer Cell Proliferation, Migration and EMT Phenotype Formation Through Sponging Mir-382. Cell Physiol Biochem 2017;42:2145-2158.

-26 Li C, Gao Y, Li Y, Ding D: TUG1 mediates methotrexate resistance in colorectal cancer via miR-186/CPEB2 axis. Biochem Biophys Res Commun 2017;491:552-557.

27 Zhang R, Jin H, Lou F: The Long Non-Coding RNA TP73-AS1 Interacted With miR-142 to Modulate Brain Glioma Growth Through HMGB1/RAGE Pathway. J cell Biochem 2018;119:3007-3016.

-28 Sanchez-Mejias A, Tay Y: Competing endogenous RNA networks: tying the essential knots for cancer biology and therapeutics. J Hematol Oncol 2015;8:30.

29 Liu XH, Sun M, Nie FQ, Ge YB, Zhang EB, Yin DD, Kong R, Xia R, Lu KH, Li JH, De W, Wang KM, Wang ZX: Lnc RNA HOTAIR functions as a competing endogenous RNA to regulate HER2 expression by sponging miR331-3p in gastric cancer. Mol Cancer 2014;13:92.

-30 Li JH, Liu S, Zhou H, Qu LH, Yang JH: starBase v2.0: decoding miRNA-ceRNA, miRNA-ncRNA and proteinRNA interaction networks from large-scale CLIP-Seq data. Nucleic Acids Res 2014;42:D92-97.

-31 Salmena L, Poliseno L, Tay Y, Kats L, Pandolfi PP: A ceRNA hypothesis: the Rosetta Stone of a hidden RNA language? Cell 2011;146:353-358.

-32 Wang H, Yu Y, Fan S, Luo L: Knockdown of Long Noncoding RNA TUG1 Inhibits the Proliferation and Cellular Invasion of Osteosarcoma Cells by Sponging miR-153. Oncol Res 2018;26:665-673.

-33 Ma F, Wang SH, Cai Q Jin LY, Zhou D, Ding J, Quan ZW: Long non-coding RNA TUG1 promotes cell proliferation and metastasis by negatively regulating miR-300 in gallbladder carcinoma. Biomed Pharmacother 2017;88:863-869.

34 Liu L, Chen X, Zhang Y, Hu Y, Shen X, Zhu W: Long non-coding RNA TUG1 promotes endometrial cancer development via inhibiting miR-299 and miR-34a-5p. Oncotarget 2017;8:31386-31394.

35 Li G, Song H, Chen L, Yang W, Nan K, Lu P: TUG1 promotes lens epithelial cell apoptosis by regulating miR421/caspase-3 axis in age-related cataract. Exp Cell Res 2017;356:20-27.

-36 Chen S, Wang M, Yang H, Mao L, He Q Jin H, Ye ZM, Luo XY, Xia YP, Hu B: LncRNA TUG1 sponges microRNA-9 to promote neurons apoptosis by up-regulated Bcl2l11 under ischemia. Biochem Biophys Res Commun 2017;485:167-173.

-37 Bash-Imam Z, Therizols G, Vincent A, Laforets F, Polay Espinoza M, Pion N, Macari F, Pannequin J, David A, Saurin JC, Mertani HC, Textoris J, Auboeuf D, Catez F, Dalla Venezia N, Dutertre M, Marcel V, Diaz JJ: Translational reprogramming of colorectal cancer cells induced by 5 -fluorouracil through a miRNAdependent mechanism. Oncotarget 2017;8:46219-46233.

-38 Yao P, Wu J, Lindner D, Fox PL: Interplay between miR-574-3p and hnRNP L regulates VEGFA mRNA translation and tumorigenesis. Nucleic Acids Res 2017;45:7950-7964.

39 Shen SQ, Huang LS, Xiao XL, Zhu XF, Xiong DD, Cao XM, Wei KL, Chen G, Feng ZB: miR-204 regulates the biological behavior of breast cancer MCF-7 cells by directly targeting FOXA1. Oncol Rep 2017;38:368-376.

$\checkmark 40$ Morel A, Baguet A, Perrard J, Demeret C, Jacquin E, Guenat D, Mougin C, Pretet JL: 5azadC treatment upregulates miR-375 level and represses HPV16 E6 expression. Oncotarget 2017;8:46163-46176.

41 Lin M, Xue XY, Liang SZ, Li YX, Lv YY, He LH, Xu KC, Zhang LF, Chen JB, Niu LZ: MiR-187 overexpression inhibits cervical cancer progression by targeting HPV16 E6. Oncotarget 2017;8:62914-62926.

42 Li X, Han J, Zhu H, Peng L, Chen Z: miR181b5p mediates TGFbeta1-induced epithelial-to-mesenchymal transition in non-small cell lung cancer stem-like cells derived from lung adenocarcinoma A549 cells. Int J Oncol 2017;51:158-168.

43 Tang BB, Liu SY, Zhan YU, Wei LQ, Mao XL, Wang J, Li LI, Lu ZX: microRNA-218 expression and its association with the clinicopathological characteristics of patients with cervical cancer. Exp Ther Med 2015;10:269-274. 


\section{Cellular Physiology Cell Physiol Biochem 2018;48:1928-1941 and Biochemistry Published online:Aungust 8, $2018 \quad \begin{aligned} & \text { DO 2018 The Author(s). Published by S. Karger AG, Basel } \\ & \text { www.karger.com/cpb }\end{aligned}$}

He et al.: The Function of TUG1-miR-142-3p-ZEB1 Axis in HCC

44 Wu G, Zheng K, Xia S, Wang Y, Meng X, Qin X, Cheng Y: MicroRNA-655-3p functions as a tumor suppressor by regulating ADAM10 and beta-catenin pathway in Hepatocellular Carcinoma. J Exp Clin Cancer Res 2016;35:89.

45 Ruan H, Yang H, Wei H, Xiao W, Lou N, Qiu B, Xu G, Song Z, Xiao H, Liu L, Zhou Y, Hu W, Chen K, Chen X, Zhang X: Overexpression of SOX4 promotes cell migration and invasion of renal cell carcinoma by inducing epithelial-mesenchymal transition. Int J Oncol 2017;51:336-346.

-46 Lu C, Shan Z, Hong J, Yang L: MicroRNA-92a promotes epithelial-mesenchymal transition through activation of PTEN/PI3K/AKT signaling pathway in non-small cell lung cancer metastasis. Int J Oncol 2017;51:235244.

47 Li Y, Lin Z, Chen B, Chen S, Jiang Z, Zhou T, Hou Z, Wang Y: Ezrin/NF-kB activation regulates epithelialmesenchymal transition induced by EGF and promotes metastasis of colorectal cancer. Biomed Pharmacother 2017;92:140-148.

-48 Zhang S, Hong Z, Chai Y, Liu Z, Du Y, Li Q, Liu Q: CSN5 promotes renal cell carcinoma metastasis and EMT by inhibiting ZEB1 degradation. Biochem Biophys Res Commun 2017;488:101-108.

49 Krebs AM, Mitschke J, Lasierra Losada M, Schmalhofer O, Boerries M, Busch H, Boettcher M, Mougiakakos D, Reichardt W, Bronsert P, Brunton VG, Pilarsky C, Winkler TH, Brabletz S, Stemmler MP, Brabletz T: The EMT-activator Zeb1 is a key factor for cell plasticity and promotes metastasis in pancreatic cancer. Nat Cell Biol 2017;19:518-529.

50 Tay Y, Rinn J, Pandolfi PP: The multilayered complexity of ceRNA crosstalk and competition. Nature 2014;505:344-352.

51 Ye K, Wang S, Zhang H, Han H, Ma B, Nan W: Long Noncoding RNA GAS5 Suppresses Cell Growth and Epithelial-Mesenchymal Transition in Osteosarcoma by Regulating the miR-221/ARHI Pathway. J Cell Biochem 2017;118:4772-4781.

\$2 Huang FT, Chen WY, Gu ZQ, Zhuang YY, Li CQ, Wang LY, Peng JF, Zhu Z, Luo X, Li YH, Yao HR, Zhang SN: The novel long intergenic noncoding RNA UCC promotes colorectal cancer progression by sponging miR-143. Cell Death Dis 2017;8:e2778.

53 Duan LJ, Ding M, Hou LJ, Cui YT, Li CJ, Yu DM: Long noncoding RNA TUG1 alleviates extracellular matrix accumulation via mediating microRNA-377 targeting of PPARgamma in diabetic nephropathy. Biochem Biophys Res Commun 2017;484:598-604. 